\title{
A Dichotomous Relationship of Intergenerational Caregiving within Elderly Care: Disruption/Disharmony and Connection/ Harmony Across the Generations
}

\section{Ya-Fen Lien ${ }^{1}$, Chun-Man Hsieh ${ }^{2}$ and Hui-Man Huang ${ }^{3 *}$}

${ }^{1}$ Department of Nursing, Meiho University, Neipu, Pingtung, Taiwan, ROC

${ }^{2}$ Department of Nursing, Tajen University, Pingtung, Taiwan, ROC

${ }^{3}$ Department of Nursing, National Quemoy University, No. 1, University Rd. Jinning Township, Kinmen County 89250, Taiwan, ROC

\begin{abstract}
In Taiwan, caregiving for older person is an intergenerational responsibility. Modern Taiwanese society faces changing family structures and increased life expectancy of older people which prolongs caregiving responsibilities, however the family relationships of intergenerational caregiving experience are not fully known. This study explored how intergenerational caregiving reconstruct relationships between offspring caregivers and their frail older people to understand how health care services can better meet family needs. We conducted a genogram and eco-map to map individual support networks and relationships. We recruited 32 family members within 12 families across three generations to participate in an individual interview, including 11 frail older people, 3 spousal caregivers, 10 adult child caregivers and 8 grandchildren. The findings demonstrated that the intergenerational caregiving experience was a dichotomous relationship between disruption/disharmony and connection/harmony. For the caregiving experiences, individuals' cultural values, beliefs, and family relationships should be considered in the context of shifting tradition, in Taiwan.
\end{abstract}

Publication History:

Received: December 01, 2021

Accepted: December 27, 2021

Published: December 29, 2021

\section{Keywords:}

Caregiving, Families, Older people, Elderly care, Taiwan

\section{Introduction}

In Taiwan, though caregiving of the frail elderly is a family responsibility, it is not restricted to two people, referred to caregiver and care recipient. Caregiving occurs across generations. With the ageing population, family relationships have become increasingly complex as the extended life expectancy of the older person has prolonged parent-child relationships [1], but family size reduced and intimate connections between different generations diminished because more educated and economic pressures increased more Taiwanese entering the workforce. Encountering Western values has reshaped cultural traditions which can create more difficulties in intergenerational caregiving. In this article, we explore such 'a contemporary phenomenon' as the intergenerational caregiving in Taiwan so as to strengthen and build new knowledge about the impact of caregiving on families to better inform policy makers and nursing practice.

Taiwan is an ageing nation. The proportion of aged $(65+)$ in Taiwan rose rapidly from 210,000 in 1951 to $1,480,000$ in $1993,3,804,000$ in 2021. From 1951 to 1993 and 2021, the ageing population represented a change from $2.5 \%$ to $7.1 \%$ and $16.2 \%$ of the total population $[2,3]$. A rapidly growing ageing population brings new challenges to health care [4]. Along with a higher proportion of the population reaching advanced age, there is a rise of older people with limited mobility, frailty or physical or mental health problems. By 2011, the frail older population in Taiwan has accounted for 407,190, 1.75\% of total population [5]. This has delivered a growth in excess of $150 \%$ over the last ten years. With the increased prevalence of older people with disabilities the demand for long-term care services including provision of formal resources such as aged care facilities and support for families in the community has steadily increased [6,7].

To meet the long-term care needs of an ageing population, there has been significant development in aged care policy with an emphasis on provision of long-term care in Taiwan [8]. Initially in Taiwan longterm care focused on institutional care but more recently shifted to community care. This change in Taiwanese government policy is underpinned by a philosophy of ageing in place with older people expected to stay in their community when getting old [9]. Although the Taiwanese government has been striving to improve long-term care services with support for the family, problems continue with many structural barriers such as complex supervisory and regulatory government agencies, as well as services inaccessible or inflexible to meet a wide range of individual needs [8].

Underpinning Taiwanese government policies is a cultural assumption that the family is responsible for caring for their frail older members. This responsibility is shaped by a cultural tradition of filial piety which provides a fundamental moral basis of caregiving systems for elders in the Chinese family [10,11]. Moreover, the allocation of family authority is built into the ties of kinship and is associated with generation, age and gender [12]. Specifically Chinese traditions enforce gendered role expectations. Accordingly whereas a son assumes major responsibilities of care for his older parents, his wife or sisters are imposed on to assume the duty of primary caregivers $[13,14]$.

Nevertheless, such traditions have been shifting. From 1950 onwards dramatic economic growth as a result of industrialization has brought considerable work and education opportunities away from the family home to urban areas [15]. Furthermore, young people have increased access to new ideas and values [15]. Specifically, encountering Western

"Corresponding Author: Dr. Hui-Man Huang, Department of Nursing, National Quemoy University, No. 1, University Rd. Jinning Township, Kinmen County 89250 Taiwan, ROC, Tel: + 886-956070508; E-mail: x2156@nqu.edu.tw

Citation: Lien YF, Hsieh CM, Huang HM (2021) A Dichotomous Relationship of Intergenerational Caregiving within Elderly Care: Disruption/Disharmony and Connection/ Harmony Across the Generations. Int J Nurs Clin Pract 8: 352. doi: https://doi.org/10.15344/2394-4978/2021/352

Copyright: (c) 2021 Lien et al. This is an open-access article distributed under the terms of the Creative Commons Attribution License, which permits unrestricted use, distribution, and reproduction in any medium, provided the original author and source are credited. 
Citation: Lien YF, Hsieh CM, Huang HM (2021) A Dichotomous Relationship of Intergenerational Caregiving within Elderly Care: Disruption/Disharmony and Connection/ Harmony Across the Generations. Int J Nurs Clin Pract 8: 352. doi: https://doi.org/10.15344/2394-4978/2021/352

Page 2 of 7

cultural values challenges Taiwanese cultural traditions [16] of family responsibility and traditional gender roles $[17,19]$. Moreover, A survey of young Taiwanese showed that only 36.5 percent of men and 19.6 percent of women believe marriage is important, a trend that academics say is key to the nation's low birthrate [20]. The resulting demographic transition has changed traditional family structures and capacity to care for their elderly. Prevalence of intergenerational households has reduced to be replaced by the small size or nuclear family, with the elderly couple or person living alone [21]. As a result, a small number of family caregivers endure every kind of burdens including increased financial pressure [22,23], adverse effect on their physical health, psychological and mental health, strain on family relationships, and interference with social life.

During past few decades, in following international agenda in addition to culture specifics, as recognizing this significance of intergenerational relationships, Taiwanese intergenerational studies have examined parents and children relationships. However, these intergenerational studies involved little work in a caregiving context across generations and understanding caregiving's influences on the intergenerational relationships. With Taiwanese culture, this study aimed to develop an understanding of caregiving experiences of offspring caregivers in contemporary Taiwan, which means the cohort is involved in caregiving for parents or grandparents, through a dyad and family-system perspective.

\section{Method}

Human life experiences are seen as multiple realities constructed through interactions between people within their sociocultural contexts [24]. Thereby we assumed that Taiwanese families are active agents engaging in the construction of their caregiving life experiences through social interactions. Their values and behaviours, influenced by sociocultural norms, could potentially affect the construction of their intergenerational caregiving.

\section{Definition of terms}

The term frail older people, older people, and the older person in this study denote the elderly care receivers who are 65 years and older. They are natural parent or grandparent of caregivers or the spouse of the caregiver. The words caregivers and offspring caregivers denote children, children-in-law, grandchildren or grandchildren-in-law of the elderly care receivers.

\section{Participant sample criteria}

After approval by the La Trobe University Human Ethics Committee (UHEC), we recruited participants to explore multiple realities from different generations within a family which comprised of the frail older person and their family caregivers including their spouse, adult children, adult children-in-law, grandchildren and grandchildren-inlaw. Based on the criteria of the Taiwanese government, frail older people had to require personal assistance in one or more key areas of Activities of Daily Living (ADL) measured by the Barthel Index, and were not cognitively impaired. Family caregivers' selection criteria included: (a) unpaid, (b) providing major or partial care and recognized by their older person and families, (c) living together with the older person, and (d) age 18 years or above. The criteria were designed to ensure that family caregivers with high levels of interaction and caregiving behaviours.

\section{Study participants}

Twelve families were recruited in this study. The researchers recognized the need for different groups in the sample to be adequately represented. These 12 families represented a diversity and typical of family structure, in Taiwan. There were four multiple-generational families, three three-generational families and five two-generational families including grandparent-child generations and grandparentgrandchild generations. The majority of families were Buddhist/Taoist whereas two were Christian. Across the 12 families, 32 participants agreed to participate in the study including 11 frail older persons, three of their spouses and 18 offspring caregivers.

Of frail older people, representing the first generation, there were ten women and two men with an age range from 70-95 years $(M=81)$. Six had no education and six completed primary school. The most common illness was cardiovascular disease followed by neurological disorders. Seven identified with high levels of physical function (BI $=60-90)$, whereas the remainder was low $(\mathrm{BI}<40)$. Three spousal caregivers were 72,75 , and 85 years of age. All were men and the primary caregivers, and had completed primary school. Generally the frail older persons and their spouses' income were low with financial support provided by government.

The majority of offspring caregivers $(n=18)$ were woman $(n=12)$, married $(n=10)$ and key caregivers $(n=8)$. Their ages ranged from 18$74(\mathrm{M}=43)$. In the second generation, there were ten caregivers: four eldest daughters-in-law, one youngest daughter-in-law, two married eldest son and three single adult children. In the third generation, eight caregivers consisted of five married grandchildren, one grandchildin-law and two single grandchildren. Nearly all offspring caregivers $(n=17)$ were educated; nine completed senior high school. Nine offspring caregivers had full-time employment, two had part-time jobs and five were unemployed. Half of offspring caregivers had low personal income. Other family income was supplemented through old-age pensions, subsidies for low income families, farm subsidies, disability pensions and housing rental subsidies.

\section{Setting and recruitment}

We conducted purposeful sampling to recruit information-rich cases that would capture multiple realities and interactions between family members [25].We recruited families from home care agencies, local care management centres and family caregiver organizations in rural and suburban areas in Taiwan. We selected those settings, because they represented a higher proportion of the elderly population and multiple-generation households.

We used four stages to recruit families. First, the researcher (YL) met the service manager to set up a meeting time with staff. At this meeting we explained the project including inclusion and exclusion criteria. Second, staff identified older people who met the selection criteria and assessed their level of competence to ensure they were able to participate. When an older person met sampling criteria, staffs provided his/her families with a recruitment pamphlet which briefly explained the purpose of this study. Third, following approve of interested families, one researcher (YL) visited their homes with a staff member to provide a copy of the information sheet \& consent form, further explain the project and address any questions. Last, if interested in participating, we arranged a suitable time to interview. We recruited multiple participants within a family mainly through 
Citation: Lien YF, Hsieh CM, Huang HM (2021) A Dichotomous Relationship of Intergenerational Caregiving within Elderly Care: Disruption/Disharmony and Connection/ Harmony Across the Generations. Int J Nurs Clin Pract 8: 352. doi: https://doi.org/10.15344/2394-4978/2021/352

Page 3 of 7

the first family member who we had contacted with, generally adult children, further negotiated participation of the older person and other caregivers. The families identified a significant caregiver person of the elderly participant in each generation.

\section{Interview Protocol and Procedure}

\section{Interview protocol}

Multiple data collection strategies included genogram, ecomapping and individual semi-structured interviews and sociodemographic data to capture clarity of the meaning data collected from different perspectives [26]. Data were collected over an eightmonth period; each generation was interviewed separately one time. The interviews were conducted in the families' homes. The interview was mainly audio-taped or collected by field notes especially elderly participants felt uncomfortable with the tape-recorder. The duration of an interview was approximately $60-90$ minutes. Few older people who tired easily spoke less than 30 minutes.

\section{Interview procedure}

Interviews began by the participant and the researcher (YL) developing the genogram and eco-map for facilitating to further explore related-issues in the following interview and to build the relationship between the researcher and the participant in a relaxed manner. The genogram and eco-map provided a simple understanding of care and support provision, as well as social and family relationships across generations [27]. As the participant described the important people in their life invariably, the caregiving stories began. Sociodemographic data collected at the conclusion of the interview allowed the researcher and the participant to have time to conclude the interview.

For the older participant the framework of the interview questions also intentionally different from the caregivers. It began with an exploration of a brief history of the elderly participant's disability. At the conclusion of this part of the interview an assessment of their level of activity was completed using the BI. The final part of the interview asked them about their experiences in managing their physical function and the role of the family in assisting them to manage activities of daily living. This arrangement of the interview schedule enabled us to gain deeper data and a better development of relationship with the older person.

\section{Data analysis}

The data analysis used grounded theory technique developed by Strauss and Corbin [28]. The rigorous and systematic strategies from ground theory enabled the complex and tangled data from multiple participants within a family to be analysed completely. We applied description and conceptual ordering of grounded theory to analyse the data collected. We analysed data first across each generation to understand the personal context, and then across the family to understand the interactive context of intergeneration caregiving by a cross section of relationships. Software NVivo (Ver.8) was used to organize and analyse content from interviews [29]. Throughout the period of doing the project, we applied a process of multiple sources of evidence, inquiry audit, peer debriefing and reflexive journal to establish the trustworthiness of the data $[26,30]$.

\section{Result}

The findings revealed that the intergenerational caregiving experience was a dichotomous relationship between intergenerational disruption/disharmony and intergenerational connection/harmony. These families encountered challenging and diverse situations in daily life. Caregiving burdens disrupted family life, but it also increased interaction and thereby connection between caregivers. As a result of divergent caregiving values, a high tension between family members which erupted. Meanwhile, families attempted to work around these differences for developing harmonious kinship.

\section{Disruption/connection}

\section{Interrupting family life}

Everyday caregiving tasks isolated caregivers from their world outside not only socially but spiritually. Caregiving tasks for the older person restricted caregivers' responsibilities required in other family roles such as parents, wives or husbands. One working woman shared:

He [her husband] always thinks I am not playing well my role as a wife, a mother and a daughter-in-law. He thinks that I get off work late and can't spend time with my family. Sometimes I feel grandparents are very old and no one knows when they will pass away. I think I need to come to look after and stay with them.

Inevitably, someone in the household had to do those tasks for the older person. Caregivers were tied down by everyday caregiving demands of the older person. The competing demands between the older person and other families affected interactions among family members and close family ties.

To care for the older person, caregivers were not without tension and concern within their families. The competing demands between work, school, housekeeping, parental nurture and eldercare contributed to the physical and psychological burden for caregivers. As one daughter-in-law said, "I'm very drained....Everything in my life. I have my own families to care for, chores to do, meals to cook....I worked during the day and had to take care of my mother-in-law at night; I am exhausted mentally and physically". This situation often happened for women. Traditional norms identified women as carers of their family. Now in modern Taiwan a woman is also expected to be a provider of financial support. As a consequence, they were sometimes in conflict on prioritizing and managing stressful demands.

The increasing longevity of the older person in Taiwan means that caring for the older person is a long-term commitment. One granddaughter reflected, "A prolonged sick parent finds no filial children at the bedside.... it's because you have to face emergencies, and you can't say no to them. You don't have back-up, but only yourself". Excessive caregiver burden from long periods of demanding caregiving challenged their filial obligation. Many felt unable to fulfill their roles according to their expectations; they experienced fear, frustration, helplessness and guilt.

\section{Family cooperation}

If families were to survive the added burdens of caregiving, there was a need for cooperation. As one daughter-in-law said, "He [brother-in-law] comes to visit his mother every day. He has a car, so he takes them to see a doctor, which is a great help." Shared family 
Citation: Lien YF, Hsieh CM, Huang HM (2021) A Dichotomous Relationship of Intergenerational Caregiving within Elderly Care: Disruption/Disharmony and Connection/ Harmony Across the Generations. Int J Nurs Clin Pract 8: 352. doi: https://doi.org/10.15344/2394-4978/2021/352

care increased interactions between offspring members and thereby the growth of family connectedness. As another daughter-in-law said, "Emotionally we are closer because we have to make contact with them [sisters-in-law] for any incident. When my grandfather-in-law's condition was good, we seldom went to wish him get-well or anything like that."

The shared difficulties of caregiving brought some families more close than before. Relationships between caregivers and care recipients enhanced and the relationships between caregivers were identified as a friendship which enabled them to share feelings. One grandchild shared,

Our relationship has improved; we're like friends. For instance, after I come home from work, my mother will tell me how grandmother is doing today. If grandmother isn't feeling well, we rush to see her and ask her 'What's wrong with you?'

Shared caregiving to improve the wellbeing of the frail older person established a link between family members; hence, intimacies and cohesions were relatively enriched.

\section{Disharmony/harmony}

\section{Family disagreements}

Harmonious relationships rested on intergenerational consensus on caregiving issues. Intergenerational caregiving involved multiple caregivers in a context of shifting cultural values. When families' values were different, conflict or pressure occurred. Lack of family agreement, the trust and supporting relationships among family members was affected.

\section{Divergent values of Life events}

They often had divergent values on medical management, leading to strained relationships in the caregiving dyad and between caregivers. One daughter-in-law caregiver described her feeling:

If she doesn't feel well and need a doctor, she will endure the pain and won't tell us because she doesn't like seeing a doctor or taking medicine.... The issue that angers me most is she starts looking for alternatives. She believes and takes drugs advertised on TV commercials or introduced by others as long as she needn't go to a doctor.

Conflicting values in what is right and proper care for elderly family distressed caregivers greatly. In older generation, traditional folk medicine was the first choice to manage illness. The next generation moved toward seeing more value in Western medical treatment accepted as the main stream in current Taiwanese society, its use was equated with best care. Failure to use "best-practice" could be equated with the family's failure to care for the older members of their family.

Some participants had double roles in a family such as be a 'daughter-in-law' and both a "grandchild'. In traditional Chinese family, system of dual caregiving responsibility had brought a lot of criticism especially from those with traditional authority in the family. Grandchild caregivers struggled with their commitment to be filial to their grandparents. They often reported unsupportive behaviors based on traditional division of family authority:

Unfortunately, there were problems in the hospital. They [medical staff] wanted to give him [older people] a tranquilizer, so they wanted family members to sign consent. We couldn't sign as it required his son to sign not grandchildren. So we had to wait for them [uncles and aunts] to sign. They wouldn't understand how we felt during the time we were waiting.

Traditional norms identified older generations as having the right to decide family matters. To be primary caregivers this grandchild generation was frustrated by the lack of power to determine caregiving requirements of the older person. In addition, they were open to criticism about the appropriateness of their caregiving actions and its consequences. This grandchild further stated,

If someone doesn't know our situation, and see the way I am taking care of him, they might say, 'What kind of care have you provided to make him sick like this? What happened to your grandfather? Haven't you attended to him?' My aunt comes home, and she says why grandfather and grandma have this and have that. How will I know? If I know, I will be a doctor and won't be what I am now.

Reassigning caregiving responsibility based on who was available or willing might have challenged traditional norms; however, the decision making power did not also follow. Such criticism from the second generation could be seen as exercising filial responsibility following traditional lines of authority. At an individual level, though it could be suggested that complaining about the quality of care assuages the aunt's guilt for not fulfilling her traditionally designated filial obligations.

\section{Traditional against neo-filial perspectives}

Traditional and new cultural norms simultaneously existed in these intergenerational families. Stress from changing traditional values with respect to caregiving responsibilities negatively affected the family relationships on different generations.

The traditional values of parent-child-relationships might dictate that children should be filial to their parents; however, it does not prevent tension between parents and children. Parental expectations of their children failed to be realized and created discord. One daughter complained about her father, "He always asks me to do extra work, and I get really upset about that and say, 'She's your own mother. What makes you feel uncomfortable when you do extra work? [for grandma]?”' Conflict was created by generational differences on values of filial piety. The young daughter saw caregiving responsibilities should be equally shared between both genders. Whereas, the father actions reflected his thoughts that decisions made by the son (man), the daughter bear the burden and heat of the caregiving responsibilities.

Tension between mothers and daughters-in-law was the most obvious issues where the responsibilities of the 'woman-in-laws' are strongly dictated by Chinese traditional norms. As one daughter-inlaw caregiver complained: "Although things my mother-in-law wanted me to do are not heavy, it was the stress. The stress was like why me, why always me." However, challenges to in-law relationships occurred as younger women encountered new values. As one caregiver described her daughter-in-law,

She [a granddaughter-in-law] seldom treats the elderly members well. For example, her grandmother [-in-law] is being cared for by me; she does not think in terms of care giving to her.... I do everything in this house. The young people do not appreciate my hard work at all. 
Citation: Lien YF, Hsieh CM, Huang HM (2021) A Dichotomous Relationship of Intergenerational Caregiving within Elderly Care: Disruption/Disharmony and Connection/ Harmony Across the Generations. Int J Nurs Clin Pract 8: 352. doi: https://doi.org/10.15344/2394-4978/2021/352

Page 5 of 7

This eldest daughter-in-law caregiver suffered ambivalent feelings between traditional and new norms. Despite her ill health, because she was unemployed, she had to accept her fate: "Well, (sigh) I have no idea what can be done. I feel this is the fate of mine, right?" Fate as defined by Buddhism intersected with the cultural responsibility dictated by Confucianism. Spiritual beliefs also infused decisions about whether to accept modern treatment options. As one daughterin-law said, "Before the gastric bleed, she couldn't walk well. We said she needed a wheelchair, but she said, 'no, no, no. If Jesus lets me be able to walk, then I walk.”

Within generation discord, following traditional roles and responsibilities according to age, generation and gender did not mean conflict did not occur. The unequal caregiving duties on siblings which families perceived created most disharmonies. One caregiver complained that her cousin did not repay through involvement in care provision but gained financial benefits from their grandparents:

I think that the person taking care of grandparent should be rewarded and the reward is the land....however, he [cousin] thought, 'I don't care, I just want to earn money and leave here'... When his parents fought and got divorced, his parents didn't want their children. So they were left to grandparents by their parents.... Now, we are grownups. We have to pay back, and we cannot be selfish and only think how much we earn.

The debate between what was right (filial piety) and just (financial reward) was played out in this family. The situation of quibbles over real property, nurturance and caregiving responsibilities repeated over time and again. Traditional roles and responsibilities were challenged especially when another member did not follow the family rules. As one eldest son said,

....I just care that it seems my mother doesn't treat me fairly. She thinks I was the oldest so I have responsibility to take care of her. For example, my father died and left a fortune. She said it was fair if three children inherited equally....Yet, she gave most part to my younger brother...

Efforts by the eldest sibling to exercise power and decide caregiving management might suggest adherence to traditional norms. However, the decisions about who should receive medical care appeared to be motivated by self-interest yet, as the young caregiver explained, "IIt is unfair], but what can I do? They [grandparents] wanted to give them [eldest grandson], and what can I say?" Traditional values dictated who would inherit but reciprocity in care provision did not follow.

Decisions within the grandchild generation to care for ageing grandparents were not always mutually agreed. Ambivalence and debate occurred between couples within families about who has caregiving responsibility. One wife reported, "To be honest, I was very upset! My thinking was that we are only his grandchildren and why us? Why not his children or his daughter-in-law to take care of him? Why skip them and we take the responsibility?" This wife saw that traditional filial obligations did not identify grandchildren as caregivers for their grandparents whereas his husband saw it was his duty to care for his grandparents as repayment for their nurturing of him. Finally, this couple divorced because a belief of traditional family wife should obey her husband's decision.

\section{Finding a meaning in caregiving}

Tension and conflict from divergent values created intergenerational disharmony but there was also potential for harmony to arise from a complex and difficult family life. Caregivers were aware that they learnt from these caregiving experiences. They discovered meaning in life. It reshaped improper behaviors and finally leaded to improved family relationships.

To consider that they might be required to care for others in the family implied an acceptance of ongoing filial responsibilities. At an individual level, with increased understanding of the situation for the older person, there was changed behavior. Caregivers' reflection on their situation brought tolerance and compassion. They also had an increased sense of caregiving responsibilities. Regardless of personal interests familism began to grow. This process was described by one grandchild:

After her strokes, I realized how terrible my behaviors were. I shouldn't have talked back to them all the time; I shouldn't have made them upset all the time.... It is common for old people not to be able to understand young people's thinking, but what they do is for the good of us even if we can't communicate to each other well. I then started to change myself.... All these make me realize I have my responsibility for them, and I should do it before it's too late....

Intergenerational connections reformed as younger generations reported reconnection across generations and sought to foster and maintain them. Accordingly, caregiving resulted in increasing interactions and intimacy through revised behaviors in the caregiving dyad. Furthermore, in assuming greater responsibility, other family relationships improved.

Caregivers were not only changing; the older person also changed. Whether it was because of their deteriorating physical health was unclear. Alternatively the families perceived there might have been a sense of appreciation of their care. In the grandchild's view, previously inappropriate behaviors of older persons changed. As one daughterin-law stated, "We treat each other with courtesy; we have no conflicts. She's dependent on me; she won't have conflict with me, right?" Finally, it created a friendship in the caregiving dyads. This caregiver further elaborated, "I will try to manage things tactically or communicate directly with my mother-in-law. We need to show our respect to the elderly members and treat them as how we treat our friends."

When they were independent, the older person always had higher authority to decide family matters. Now, being a dependant meant diminished power and the need to show some respect for younger people. This change improved intergenerational relationships as families compromised and reached agreement and as a consequence better relationships and friendships between older persons and caregivers blossomed.

\section{Discussion}

Throughout this study it is demonstrated that the families' lives were affected by caregiving responsibilities. Conflict based on divergent values and interrupted family life harmed family relationships, whereas family cooperation and changing behaviors through learning and finding meaning in life improved intergenerational relationships.

The health status of older persons, day-to-day caregiving tasks and competing roles were primary stressors that further led to secondary stressors on role strains such as economic hardships, family conflict and constraints on social and family life [22,31-32]. Day-to-day caregiving tasks created psychological distress which was expressed 
Citation: Lien YF, Hsieh CM, Huang HM (2021) A Dichotomous Relationship of Intergenerational Caregiving within Elderly Care: Disruption/Disharmony and Connection/ Harmony Across the Generations. Int J Nurs Clin Pract 8: 352. doi: https://doi.org/10.15344/2394-4978/2021/352

with a mixture of negative emotions such as frustration, anxiety, fear anger, guilt, sadness, and depression [32-35].

However, each day they faced multiple family and social roles and responsibilities combined with a lack of access to adequate internal and external support. Early research by Pearlin, Mullan, Semple, and Skaff [36] noted that the caregiver role was intensive, relentless and ongoing and can be inhibited or supported by family networks and social support. Without available manpower and finance to purchase services, children and grandchildren often faced competing needs for themselves and their own family along with caregiving demands of the older person. Among intergenerational studies in caregiving, only one American study identified caregiver fatigue as an issue [33]. In that study, middle aged grandchildren cared for a dying grandparent in a hospice setting with a known endpoint. This does not help to explain the experiences of offspring caregivers in the community across generations and age, as well as the influence of macro level cultural and social norms.

The second difference from other findings was caregivers often had fears of deterioration of the health status of the older person which were linked to the family's capacity to care for them. Over time the older persons' capabilities were reducing, whereas their care needs escalated [34]. Caregiving tasks became more and more unpredictable and unplanned. This continual fluctuation between wellness and ill health is common in the elderly with multiple comorbidities [37]. Its impact on the family was significant. Living with future uncertainty built up fearful feelings, not only concern for the older person but also whether caregivers would actually be capable of continuing their caregiver roles. It challenged their moral commitment to adhere to filial obligations. The breakdown of family networks and social support amplified this distress. A constant and undirected fear of the older person's falling and its serious consequence led to intensive and vigilant care to prevent their occurrence $[38,39]$. Families became ambivalent about encouraging older persons to exercise to maintain their health or to promote less mobility to avoid falls which might contribute to their functional decline.

In spite of all the family caregiving difficulties experienced, there were positive affirmations in intergenerational caregiving identified by the caregivers. The rewards included: acquiring caregiving knowledge and skills, finding meaning in life, and positive self-affirmation, as well as enhanced family relationships. Caregiving was learning and relearning process $[32,35,40]$. Adult child and grandchild caregivers learnt new skills from "doing".

Caregiving was seen as a personal turning point for some caregivers. For them they saw that caregiving facilitated personal growth and reformulated their self-identity $[32,34]$. Through new meaning in their life previously held beliefs and behaviours were reshaped. Che et al. identified caregiving as a self-empowerment process, where despite the negative impact of caregiving, the experience also inspires an "inner awareness" that helps them examine and manage their caregiving encounters [13, p.212]. In this study, some caregivers, especially grandchildren, witnessing the deteriorating physical function of the frail older person led them to recognize the family relationships; therefore, they felt compelled to change their behaviors to become caring, tolerant and responsible. These changes suggest that family caregivers were learning to conform and accept traditionally held values and responsibilities. This however was not always the situation. Some daughters-in-law moved toward individualism to consider their own interests, and expected other families to share the caregiver duty [22]. They also expected older persons to contribute to their own self-care to promote independence and better quality health.

Caregivers experienced rewards of self-affirmation from caregiving which were similar to other findings $[22,31,35,38]$. The exchange of caregiving experiences in their social network in which knowledge and skills were shared increased the feeling of usefulness and self-worth of caregivers. Moreover, because caregivers successfully fulfilled their multiple duties related to employment, housework and/or caregiver, a rise of personal satisfaction, self-reliance and self-esteem could occur $[22,35,38]$

Also, when family members strongly commit to their family roles and obligations, there is a higher level of exchange of resources, sentiments and interactions [41,42]. It is argued that caregiving brings improvement to interpersonal relationships in the caregiving dyad and between offspring caregivers [34,40]. Differently, this study represented a changing relationship across three generations. Previous inappropriate behaviors of families were adjusted because of a new meaning of life. Although traditional norms that bestowed authority to older generations could result in conflict with other generations, being dependent resulted in older people relinquishing control of their traditional power. It also appeared that older-generation offspring moved toward individualism, and younger-generation offspring moved toward familism. With equal allocation of power and caregiving duty the tension in the caregiving dyad decreased. There was a blurring of authoritarian boundaries. Cooperation between families led to improved quality of care and new intimacy, from which some new friendships developed to manage the caregiving life [43].

\section{Study Limitation and Future Research}

This paper provides information on the tasks of intergenerational caregiving families with frail older members in Taiwan. The findings demonstrated that the intergenerational caregiving experience was a dichotomous relationship between disruption/disharmony and connection/harmony, in Taiwan. Because of the small sample size and sampling criteria, the findings are not representative of older adults living alone. Individuals' cultural values, beliefs, and family relationships should be considered in the context of shifting traditions.

\section{Competing Interests}

The authors declare that they have no competing interests.

\section{References}

1. Bengtson VL (2001) The burgess award lecture: Beyond the nuclear family: The increasing importance of multigenerational bonds. Journal of Marriage and Family 63: 1-16.

2. Ministry of the Interior (2007) Ten-year plan for long-term care in Taiwan: Flagship plan for big warm social welfare scheme. Taipei: Executive Yuan.

3. Department of Statistics (2021) Statistical yearbook of interior: Population by Age.

4. Dhar VE (2011) Transnational caregiving: Part 1, caring for family relations across nations. Care Manag J 12: 60-71.

5. Department of Statistics (2011) Statistical yearbook of interior: The disabled population by age and grade.

6. Clinco JB (2010) Direct caregivers association: An option for a rapidly growing aging population? The Care of the Uninsured in America. New York: Springer. 
Citation: Lien YF, Hsieh CM, Huang HM (2021) A Dichotomous Relationship of Intergenerational Caregiving within Elderly Care: Disruption/Disharmony and Connection/ Harmony Across the Generations. Int J Nurs Clin Pract 8: 352. doi: https://doi.org/10.15344/2394-4978/2021/352

7. Meijer CAM, Majer IM, Koopmanschap MA, Baal PH (2012) Forecasting lifetime and aggregate long-term care spending: Accounting for changing disability patterns. Med Care 50: 722-729.

8. Tsai CY (2010) Taiwanese long-term care services. Community Development Journal 129: 410-425.

9. Chiang LY (2009) Aging society: Aging in place. Community Development Journal 125: 195-210.

10. Jie Z (2003) Collectivism or individualism: An analysis of Chinese and Taiwanese interactive culture. Taiwan in the twenty-first century. Lanham: University Press of America.

11. Van Norden CR (2002) Introduction. Confucius and the analects. New York: Oxford University Press.

12. Hwang KK (2001) The deep structure of Confucianism: A social psychological approach. Asian Philosophy 11: 179-204.

13. Che HL, Yeh ML, Wu SM (2006) The self-empowerment process of primary caregivers: A study of caring for elderly with dementia. J Nurs Res 14: 209218

14. Hsu HC, Shyu YL (2003) Implicit exchanges in family caregiving for frail elders in Taiwan. Qual Health Res 13: 1078-1093.

15. Gold TB (2008) Taiwan society at the fin de siècle. The politics of modern Taiwan: Nationalism and national identity. Oxon: Routledge.

16. Zhiyuan C (2003) Education in Taiwan. Taiwan in the twenty-first century Lanham: University Press of America.

17. Ku Y (2008) Feminist activism within bureaucracy: Process of formulating and implementing regulations governing the protection of women's rights in Taipei. Women's Studies International Forum 31: 176-185.

18. Department of Statistics (2011) Statistical yearbook of interior: Population by Age.

19. Yang FI (2007) Beautiful-and-bad woman: Media feminism and the politics of its construction. Feminist Studies 33: 361-425.

20. Hui-ju C, Madjar K (2021) Low interest in marriage linked to birthrate.

21. Syue CT (2008) Family transition and living arrangements for elders: Present and future. Community Development Journal 121: 47-56.

22. Hashizume $Y$ (2010) Releasing from the oppression: Caregiving for the elderly parents of Japanese working women. Qualitative Health Research 20: $830-844$

23. Østbye T, Chan A, Malhotra R, Kothalawala J (2010) Adult children caring for their elderly parents: Results from a national survey of ageing in Sri Lanka. Asian Population Studies 6: 83 - 97.

24. Burr V (2003) Social constructionism. London: Routledge.

25. Patton MQ (2014) Qualitative Research and Evaluation Methods. California: Sage.

26. Yin RK (2014) Case Study Research: Design and Methods. California: Sage.

27. Iversen RR, Gergen KJ, Fairbanks RP (2005) Assessment and social construction: Conflict or co-creation? British Journal of Social Work 35: 689708.

28. Corbin J, Strauss A (2015) Basics of Qualitative Research: Techniques and Procedures for Developing Grounded Theory. Thousand Oaks, CA: Sage.

29. QSR International (2009) NVivo 8 Doncaster, Vic, Australia.

30. Lincoln YS, Guba EG (1985) Naturalistic inquiry. Sage, California.

31. Escandón S (2006) Mexican American intergenerational caregiving model West J Nurs Res 28: 564-585.

32. Fruhauf CA, Jarrott SE, Allen KR (2006) Grandchildren's perceptions of caring for grandparents. Journal of Family Issues 27: 887-911.

33. Boquet JR, Oliver DP, Wittenberg-Lyles E, Doorenbos AZ, Demiris G, et al (2011) Taking care of a dying grandparent: Case studies of grandchildren in the hospice caregiver role. American Journal of Hospice \& Palliative Medicine 28: 564-568.

34. Campbell LD (2010) Sons who care: Examining the experience and meaning of filial caregiving for married and never-married sons. Can J Aging 29:7384.

35. Fruhauf CA, Orel NA (2008) Developmental issues of grandchildren who provide care to grandparents. Int J Aging Hum Dev 67: 209-230.

36. Pearlin LI, Mullan JT, Semple SJ, Skaff MM (1990) Caregiving and the stress process: An overview of concepts and their measures. Gerontologist 30 : 583-594.

Int J Nurs Clin Pract

ISSN: 2394-4978
37. Lunney JR, Lynn J, Foley DJ, Lipson S, Guralnik JM, et al. (2003) Patterns of functional decline at the end of life. JAMA 289: 2387-2392.

38. Faes MC, Reelick MF, Joosten-Weyn Banningh LW, Gier M, Esselink RA, et al. (2010) Qualitative study on the impact of falling in frail older persons and family caregivers: Foundations for an intervention to prevent falls. Aging Ment Health 14: 834-842.

39. Stockwell-Smith G, Kellett U, Moyle W (2010) Why careers of frail older people are not using available respite services: An Australian study. J Clin Nurs 19: 2057-2064.

40. Perry J (2004) Daughters giving care to mothers who have dementia: Mastering the 3 R's of (re)calling, (re)learning, and (re)adjusting. Journal of Family Nursing 10: 50-69.

41. Bengtson VL, Roberts EL (1991) Intergenerational solidarity in aging families: An example of formal theory construction. Journal of Marriage and Family 53: 856-870.

42. Roberts REL, Bengtson VL (1990) Is intergenerational solidarity a unidimensional construct? A second test of a formal model. J Gerontol 45 S12-S20.

43. Fischer LR (1986) Linked Lives: Adult Daughters and Their Mothers. Harper and Row, New York. 